\title{
[gw22-e0139] THE CLINICAL MANIFESTATION OF SUBJECTS WITH RHABDOMYOLYSIS INDUCED BY RED
} SWAMP CRAWFISH IN CHINA

Jia Enzhi, Chen Xufeng, Zang Xiaoling, Yang Zhijian, Zhang Jinsong The First Affiliated Hospital of Nanjing Medical University

10.1136/heartjnl-2011-300867.727

Objective The objective of this report describes the clinical and epidemiologic characteristics of the 17 patients with confirmed cases of rhabdomyolysis induced by red swamp crawfish.

Methods Between August 10, 2010 and August 31, 2010, 17 patients were admitted to the department of emergency, the first affiliated hospital of Nanjing Medical University with a history of consumption of red swamp crayfish, and the patients were diagnosed as having rhabdomyolysis. All 17 patients had serum creatine kinase and myoglobin levels obtained or serially monitored either on presentation to the emergency department or after hospital admission.

Results The median peak CK level was 9026.0 IU/1, which was detected on the 13-16th h. The median peak blood myoglobin level was $1000 \mu \mathrm{g} / \mathrm{l}$, which was detected on the 5-12th $h$. The creatine kinase and myoglobin had normalised on the 121-144th and 69-72th h. After treatment with aggressive hydration and alkalinisation, none of the patients had acute renal failure. All recovered completely.

Conclusions The earlier diagnosis and progressive treatment are essential for the prognosis of the subjects with rhabdomyolysis, and the mechanisms of rhabdomyolysis caused by red swamp crawfish needs further study. 